\title{
PSICONCOLOGIA: PERSPECTIVA TEÓRICA E METODOLÓGICA COMPORTAMENTAL
}

Suzane Schmidlin Löhr ${ }^{*}$ Cloves Amisses Amorim

\section{RESUMO}

O presente estudo reflete dados da literatura, relacionando os avanços no tratamento do câncer com conhecimentos da Psicologia segundo o referencial teórico comportamental. Aponta as origens da Psiconcologia (interface da Psicologia e Oncologia), observando que a preocupação em evidenciar fatores etiológicos e de manutenção das doenças é antiga, enquanto que o estabelecimento de relação entre doença e estilos de vida é recente. Pesquisas enfocando o estudo de aspectos psicológicos do câncer atrelam-se, ora à Medicina Comportamental, ora à Psicologia da Saúde. Na seqüência, analisa-se a influência de fatores psicossociais na adoção de comportamento de prevenção na área do câncer, bem como estratégias comportamentais de intervenção frente ao paciente

Doutoranda da USP em Psicologia Clínica, Professora do Departamento de Psicologia da Universidade Federal do Paraná.

** Doutorando em Psicologia na Espanha, Professor de Psicologia da Pontifícia Universidade Católica do Paraná. 
com câncer ou a sua comunidade. O estudo enfoca a intervenção psicológica nas diferentes fases do tratamento médico: prevenção, diagnóstico, tratamento, reabilitação, pós-tratamento ou acompanhamento na fase terminal através de cuidados paliativos.

A proposta de buscar cientificamente relações entre fatores psicossociais e a incidência, evolução e remissão do câncer é nova, segundo GIMENES (1994). No entanto, a autora aponta como esta questão esteve presente desde o tempo da medicina e filosofia pré-cartesianas.

A preocupação recente, quanto à qualidade de vida dos indivíduos com doenças crônicas, tem estimulado pesquisas visando compreender a influência de diferentes fatores tanto na eclosão como na manutenção e tratamento das doenças, pois da adequada compreensão e delimitação destes aspectos podemos extrair formas de prevenção, bem como tratamentos mais eficazes e menos penosos.

A Oncologia e a Psicologia são áreas de conhecimento tradicionalmente estruturadas e sedimentadas. A interface delas, a Psiconcologia, é recente, estando aqui no Brasil em fase inicial. Sua proposta de análise envolve "a identificação do papel de aspectos psicossociais, tanto na etiologia quanto no desenvolvimento da doença, a identificação de fatores de natureza psicossocial envolvidos na sua prevenção e reabilitação, bem como vem incentivar a sistematização de um corpo de conhecimento que possa fornecer subsídios tanto à assistência integral do paciente oncológico e de sua família, como também à formação de profissionais de Saúde envolvidos com o seu tratamento" GIMENES (1994).

Quando a comunidade científica começa a perceber que tanto o surgimento do câncer quanto a sua manutenção ou remissão vão além da natureza biomédica do problema, nasce a Psi- 
concologia. É no âmbito da Psiconcologia que vamos encontrar espaço para analisar o câncer sob a vertente da Psicologia, com suas múltiplas abordagens. É fundamental para o avanço do conhecimento que cada profissional possa mergulhar em sua área de estudos e, a partir de sua perspectiva, contribuir para a construção de um conhecimento mais amplo.

Pretendemos, neste momento, tazer uma análise dos aspectos psicológicos relacionados ao câncer segundo o referencial comportamental. No enfoque comportamental tem-se como núcleo da análise o comportamento, o qual foi definido por SKINNER (1991) como uma RELAÇÃO entre o meio e o organismo (não é nem o meio e nem o organismo, mas a relação entre eles).

Partindo da definição dada por SKINNER (1991) para "comportamento", procuraremos entender o câncer aos olhos da abordagem comportamental. Tal tarefa remete-nos às pesquisas oriundas da Medicina Comportamental, a qual é considerada um dos campos de atuação e pesquisa decorrente do modelo biopsicossocial.

A Medicina Comportamental, segundo SCHWARZ e WEISS (1978), floresceu nos anos 70, desenvolvendo-se a partir dos pressupostos da Psicologia Comportamental que envolvem principalmente os paradigmas de condicionamento operante e condicionamento respondente e da fisiologia experimental (efeitos da emoções sobre o funcionamento do organismo).

Já outros autores, referindo-se ao campo de atuação que engloba o estudo do câncer, empregam nomenclaturas distintas, como Psicologia da Saúde (MATARAZZO, 1980) ou Medicina Psicossomática (LIPOWSKI, 1986). Na verdade, conforme já apontado por GIMENEZ (1994) e SARAFINO (1991) apud CARVALHO (1994) sob o ponto de vista da organização, Psicologia da Saúde, Medicina Comportamental e Medicina Psicossomática são distintas. Porém quanto à visão de saúde e doença como resultante da inter-relação entre fatores biológicos, psicológicos e sociais há 
concordância entre elas, contribuindo assim para o fortalecimento de um modelo Biopsicossocial.

Uma vez definida ou discutida a perspectiva que ora se denomina Psicologia da Saúde, ora Medicina Comportamental, impõe-se uma questão conceitual acerca da existência de um modelo que delimite esta perspectiva. Emílio Ribes IESTA (1990) apresenta alguns pressupostos de um modelo psicológico para esta análise. Seu modelo tem como objetivo a delimitação teórica e conceitual dos problemas de saúde e doença. O autor alerta para o fato de que conhecer o processo biológico de uma doença e as condições sociais de sua epidemiologia não são suficientes, faz-se necessário ir além, aplicando este conhecimento na geração de medidas efetivas a serem implementadas no dia-a-dia das pessoas.

Ribes INESTA (1990) propõe em seguida um esquema incluindo dois tipos diferentes de categorias descritivas : os processos psicológicos, que vinculam condições do organismo com ações do indivíduo dentro de um meio sócio-cultural, e os resultados ou conseqüências de tais processos, referentes a características funcionais do comportamento, a vulnerabilidade do organismo e seus efeitos na produção-prevenção de doença, assim como comportamentos associados à alteração biológica.

$\mathrm{O}$ autor acima identifica três tipos de problemas psicológicos relacionados à doença física biológica: 1. condições psicológicas como predisposições ou propensão comportamental, derivadas de contingências biológicas; 2 . comportamentos normalmente associados às doenças biológicas que ocorrem na ausência de doenças, como conseqüência dos efeitos puramente instrumentais em nível social; 3. comportamentos que surgem em reação e como conseqüência de uma doença biológica.

Sendo a proposta do presente artigo discorrer sobre um tipo específico de doença física, o câncer, julgamos oportuno conhecêla um pouco melhor. Câncer é uma denominação geral que inclui 
mais de 100 tipos de doenças como, por exemplo, as leucemias, tumores, osteosarcoma e outras, sendo que o fator que as aproxima e inclui no mesmo grupo é o fato de em todas elas ocorrer um crescimento anormal e descontrolado das células do organismo. Questões acerca do que determina este crescimento anormal e desordenado de células há muito vêm preocupando pesquisadores. Numa vertente organicista, a busca de respostas remete a estudos da determinação genética.

Por outro lado, encontramos citações como as de JENKINS (1990): "Toda doença humana difundida deve sua prevalência, no todo ou em parte, a processos sociais ou comportamentais. Estes processos podem estar envolvidos na sua patogênese, transmissão e/ou remediação".

Nesta linha de análise, GORAYEB (1990) refere que se todos os processos sociais e comportamentais podem potencialmente ser mudados, há também que se levar em conta a possibilidade de se intervir em prol da edificação específica de comportamentos de risco dos indivíduos, melhorando sua consciência sobre as relações comportamento e saúde, sua consciência quanto a seus direitos na área da saúde e sua condição de vida.

Cada vez mais fala-se do homem como um ser biopsicossocial. Segundo tal concepção, não é possível buscar um único fator etiológico capaz de desencadear o câncer. Porém, quanto mais as diferentes áreas de conhecimento se unirem, mais possibilidades de prevenção e cura serão abertas, o que se torna particularmente importante se for observado que o cancer é hoje incluído entre uma das quatro maiores causas de mortalidade no Brasil.

BAYES (1991), ao afirmar estratégias de prevenção do câncer, informa que um processo canceroso pode ser considerado como o resultado da intervenção de quatro tipos de variáveis: a) uma base biológica, produto e herança e uma história individual concreta; b) elementos carcinogênicos procedentes do ambiente, tanto do ambiente natural (irradiações, vírus, etc.), como do criado 
pela ação do homem (contaminação, substâncias ou radiações próprias de alguns trabalhos, etc.); c) elementos carcinogênicos introduzidos pelo indivíduo no seu organismo ou produzidos como conseqüência de seu estilo de vida - alimentos, cigarro, álcool, falta de exercícios físicos, etc.; d) fatores psicológicos capazes de produzir estados de imunodepressão.

Ribes IÑESTA (1990) sugere que um plano eficaz de prevenção de câncer deveria levar em conta os quatro tipos de variáveis, investigando-as em profundidade a fim de propor estratégias de intervenção. Ressalta serem as campanhas informativas necessárias, porém não suficientes, pois o fato de uma pessoa possuir um conhecimento não é garantia de que seu comportamento será modificado.

Alguns obstáculos podem ser apontados como possíveis responsáveis pela dificuldade em se adotar comportamentos de prevenção aos eventos causadores do câncer: 1 . o caráter prazeroso da maior parte das estimulações que se seguem aos comportamentos nocivos, assim como a imediatez do efeito. Observese a sensação de relaxamento experimentada após fumar um cigarro, o sabor agradável, que acompanha a ingesta de alimentos, o bronzeado e os olhares que suscita, etc. Notamos aqui a imediaticidade do reforço positivo, enquanto que a punição é apenas uma probabilidade e distante temporalmente da emissão do comportamento; 2 . o grande período de tempo entre a prática do comportamento nocivo e o aparecimento da enfermidade em seu estado clínico diagnosticável dificultam o estabelecimento da relação causal; 3. o fato de que muitos estímulos carcinogênicos proporcionam quase sempre uma satisfação real e imediata enquanto o surgimento do câncer é apenas uma probabilidade, exemplifica a ação de esquemas de reforçamento intermitentes, os quais têm muito mais força na manutenção do comportamento, visto que esporádicos, conforme já demonstrado experimentalmente por vários autores, e apontado por KELLER e SCHOENFELD (1974). 
Para BAYES (1987), a análise experimental do comportamento mostra que, em situações como as descritas acima, o sujeito elegerá o prazer certo e imediato, apesar das terríveis conseqüências a longo prazo que sua ação poderá acarretar.

Assim, uma ação preventiva eficaz deveria considerar simultaneamente objetivos em três níveis, ou seja: buscar inicialmente alterações ambientais visando minimizar os agentes carcinogênicos, ao mesmo tempo em que, indo além, propusesse modificação de hábitos de vida e também alteração das expectativas e respostas emocionais dos indivíduos frente à doença.

Outra frente de investigação do câncer, decorre dos avanços da psiconeuroimunologia. Estudos multidisciplinares enfocando a interação entre stress, conduta, funções endócrinas, sistema nervoso e sistema imunológico (LESHAN, 1992; MARIN et. al., 1994; MOSCOSO e OBLITAS, 1994) têm permitido entender que certos processos comportamentais são capazes de influenciar funções do sistema imunitário e que o estado imunológico tem influência significativa sobre a conduta.

O sistema imunitário é um dos mecanismos de vigilância e defesa, cuja responsabilidade é combater as doenças causadas por microorganismos e outros agentes estranhos. Estudos de animais em laboratório mostraram que agentes estressores podem suprimir as respostas de defesa do sistema imunitário através da redução de linfócitos. Em nível humano, estudos empíricos de vários autores, compilados por MOSCOSO e OBLITAS (1994), indicam que eventos estressantes como cirurgia, exames acadêmicos e falta de sono associam-se com decréscimo na concentração de linfócitos e células T no sistema imunitário.

Pesquisas como as acima citadas têm contribuído à compreensão das complexas interações entre variáveis psicológicas e saúde-doença, lançando questões pertinentes à mediação das emoções, stress e aspectos cognitivos sobre o sistema imunológico, tão bem sintezadas por MOSCOSO (1994), ao referirem-se a inúmeros trabalhos de outros autores nesta área. 
A relação entre stress e doença não é simples. Existe uma série de variáveis disposicionais, de personalidade e estilo de enfrentamento, percepção e avaliação cognitiva, suporte social e recursos pessoais à disposição, os quais cumprem papel importante dentro do processo saúde-doença.

Sintetizando, podemos dizer que o câncer implica uma alteração biológica envolvendo multiplicação desordenada e rápida de células anormais, podendo decorrer de aspectos genéticos e/ou ambientais, porém sujeito a influências pessoais através do grau de controle do sistema imunitário. Por outro lado, o grau de controle do sistema imunológico está diretamente relacionado a características de personalidade (concebendo personalidade como as formas do indivíduo lidar com as situações que a vida lhe impõe), as quais decorrem da história de aprendizagem do indivíduo.

O stress está presente na vida de todos os indivíduos, variando em intensidade em diferentes períodos e de acordo como o contexto. Observamos, no entanto, que cada indivíduo pode manifestar respostas diversas frente a uma mesma situação de estímulo, caracterizando diferentes estratégias de enfrentamento ao stress. A forma de lidar com o stress influencia em diferentes graus o sistema imunitário, abrindo, assim, mais ou menos espaço para o surgimento de alguma doença que até então pudesse estar sob controle desse sistema.

Até aqui estivemos discutindo como fatores psicológicos podem influenciar no aparecimento do câncer, ou contribuir para um melhor ou pior prognóstico. Compreender estes mecanismos é crucial para que a psicologia possa desenvolver estratégias visando auxiliar as pessoas que se deparam com um quadro de doença grave e crônica como o câncer.

$\mathrm{Na}$ atualidade, vem crescendo o número de psicólogos que se dedicam ao trabalho junto aos pacientes com câncer, o que é um fator positivo e tem ampliado os recursos de intervenção. Seguindo o referencial teórico comportamental, citaremos algumas estratégias que vêm sendo empregadas. 
O primeiro âmbito de intervenção psicológica seria a prevenção psicológica, a qual foi abordada com maiores detalhes no início do presente estudo.

Se as estratégias de prevenção não forem suficientes e a doença se faz presente, o psicólogo pode atuar junto ao paciente em tratamento médico, bem como junto a seus familiares e à comunidade.

Neste momento, as intervenções serão diferenciadas de acordo com a etapa do tratamento médico. Assim, quanto ao Diagnóstico, podemos incluir programas de prevenção secundária, cuja meta seja a identificação precoce do câncer, o que eleva as possibilidades de sucesso do tratamento. Se concepções errôneas com potencial desencadeador de esquiva forem trabalhadas junto à população, mais pessoas engajar-se-ão na identificação precoce do câncer, aumentando suas chances de cura.

$\mathrm{Na}$ fase do Tratamento faz-se necessário constatar o estilo comportamental do indivíduo, pois há indivíduos que, frente à doença, desenvolvem a crença de que nada podem fazer para alterar o seu destino, ficando à mercê do que lhes venha acontecer. Tal quadro foi definido por SELLIGMAN (1975) como desamparo aprendido, estando o indivíduo sob um locus de controle externo. FONT (1994) afirma haver íntima relação entre "locus de controle" e qualidade de vida. Segundo o autor, os pacientes com pior qualidade de vida são aqueles que valorizam seus dias como negativos, sentem medo e preocupação por seu estado de saúde e apresentam baixa pontuação de locus de controle interno.

Ainda em relação ao tratamento, ASENSIO e CARULLA (1990) apresentam os sintomas de maior freqüência no tratamento de câncer, e a metodologia de intervenção frente a eles. Para os autores, o tratamento apropriado para os sintomas de náuseas e vômitos seria o relaxamento, a visualização e a dessensibilização sistemática. Nos casos de dor as técnicas operantes (combinação de técnicas de reforço positivo, modelagem, contrato de contin- 
gências) podem ser úteis, fazendo, por exemplo, com que o paciente discrimine a sua dor quanto à intensidade, localização e funcionalidade, permitindo uma medicação analgésica mais adequada e na dosagem apropriada. Em se tratando de insônia, as técnicas de terapia cognitivo-comportamental, correção de concepções errôneas e relaxamento têm sido utilizadas, visto que permitem ao paciente analisar de forma mais realística os seus medos, o que muitas vezes constitui um fator a dificultar o descanso, sendo que o relaxamento faz com que o paciente aprenda a manejar suas tensões favorecendo assim o aparecimento do sono.

Outras técnicas como a distração e automonitoria podem auxiliar no combate aos efeitos colaterais do tratamento, bem como no controle da dor e dos vômitos. Nossa experiência clínica tem mostrado que, quando fica o tempo todo atento apenas ao correr do líquido quimioterápico em sua veia, o paciente apresenta maiores respostas aversivas do que quando, neste período de espera, envolve-se em outras atividades que sejam viáveis, como, por exemplo, ouvir histórias contadas pela mãe.

Cabe aqui ressaltar o que se poderia denominar a luta pelo "eu perdido": As mudanças decorrentes do tratamento desencadeiam no indivíduo alterações em sua aparência física, que podem influenciar na sua auto-estima, as quais precisam ser adequadamente analisadas, de forma a não impedirem o fluxo natural de vida daquela pessoa.

A reabilitação é considerada um dos aspectos relevantes da intervenção, pois o fato de terminar o tratamento pode precipitar uma série de ansiedades no paciente frente à perda de apoio, medo de recidiva sem medicação, etc. Quando o tratamento implicou alguma intervenção mutilante, mais elementos estão em jogo, como a mudança na auto-imagem, as dores sentidas, a adaptação a uma nova forma de vida, já que o indivíduo precisa adaptar-se a esta nova imagem de si. Voltar ao ritmo de vida anterior à doença 
pode ser uma tarefa árdua, na qual a intervenção profissional fazse necessária, por meio da análise das condições atuais, das estratégias de enfrentamento de que o cliente dispõe, bem como das suas cognições acerca desse retorno.

BLASCO (1994) ressalta que é necessário tomar consciência da necessidade de um enfoque não atomista que valorize o paciente como pessoa e não como um sujeito submetido ao curso das leis de aprendizagem autodeterminadas, cadeias de estímulos apenas. É preciso, portanto, ir além da perspectiva de laboratório que considera o "fenômeno" isolado. Devemos ter em conta: estágio/fase da doença, objetivo do tratamento, expectativas do paciente, qualidade de vida, relação paciente/equipe, entre outros.

$\mathrm{O}$ atendimento ao Paciente Terminal é um dos pontos mais delicados da intervenção, pois quando o retorno à saúde não é possível, a qualidade do tempo de vida que resta passa a ser o ponto central. Assim, com respeito aos cuidados paliativos, COMAS (1991) propõe que, além da dignidade da pessoa humana, é preciso que alguns objetivos sejam claros e compartilhados. Reduzir a solidão do paciente, aumentar sua autonomia e autocontrole, manejar a depressão podem diminuir o stress e os efeitos da doença.

Concluindo, percebe-se que a reflexão até aqui desenvolvida aponta o quanto a análise psicológica embasada no modelo teórico-comportamental pode contribuir para desvendar uma série de variáveis relacionadas com um quadro nosológico orgânico. Esta análise fortalece a visão monista de homem, mostrando que para a sua compreensão é preciso vê-lo como um todo em que aspectos biológicos, psicológicos e sociais estão em contínua iterrelação.

A análise comportamental permite também o desenvolvimento de novas formas de intervenção, as quais podem propiciar prevenção psicológica primária, bem como melhorar a qualidade de vida durante as diferentes etapas do tratamento médico. 


\section{REFERÊNCIAS BIBLIOGRÁFICAS}

1 ASENSIO, X. P.; CARULLA, L. S. Terapia de conducta en oncologia. C. Med. Psicosom., n. 13, 6-19, 1990.

2 BAYES, R. La prevención del câncer. Jano : Medicina y Humanidades, v. 33, n. 783, p. 45-55, 1987.

3

- Psicologia oncológica. Barcelona: Martinez Roca, 1991.

4 BLASCO,T. La nausea y el vómito asociados a la quimioterapia: uma reflexão sobre a investigação básica e aplicada. Anuário de Psicologia, n. 61, p. 51-55, 1994.

5 CARVALHO, M. M. J. (Org.). Introdução à psiconcologia. São Paulo: Editorial Psy, 1994.

6 COMAS, J. Objetivos concretos da equipe de cuidados paliativos. Madrid, 1991. (mimeo). Não publicado.

7 FONT, A. Cáncer y calidad de vida. Anuário de Psicologia, n. 61, p. 41-50, 1994.

8 GIMENES, M. G. Psiconcologia : definição, foco de estudo e intervenção. In: CARVALHO, M. M. (Org.). Introdução à psiconcologia. São Paulo : Editorial Psy, 1994.

9 GORAYEB, R. Medicina comportamental : a ação do psicólogo na prevenção, tratamento e reabilitação de doenças orgânicas. In: REUNIÃO ANUAL DE PSICOLOGIA, 20., 1990, Ribeirão Preto. Anais. Ribeirão Preto : Sociedade Brasileira de Psicologia, 1990.

10 KELLER, E.; SCHOENFELD, W. Princípios de Psicologia. São Paulo : EPU, 1974.

11 LESHAN, L. O câncer como ponto de mutação. São Paulo: Sumus, 1992. 
12 LIPOWSKI, Z. J. Psychosomatic medicine - past and present. Canadian of Psychiatry, v. 31. p. 2-21, 1986.

13 MARÍN, J.; TEROL, M. C.; ROIG, S. L.; PASTOR, M. A.; MASSUTI, B. Predictores del afrontamiento en pacientes oncológicos. Ansiedad y Estrés, p. 113-119, 1994.

14 MATARAZZO, J. D. Behavioral health and behavioral medicine: frontiers for a new health psychology. American Psychologist, p. 807-817, 1980.

15 MOSCOSO, M.; OBLITAS, L. Hacia una Psicologia de la salud en el ano 2000: Retos Y promesas. Psicologia contemporânea, v. 1, n. 1,1994.

16 RIBES E. R. Psicologia y salud : un análises conceptual. Barcelona : Martins Rocca, 1990.

17 SCHWARTZ, G.; WEISS, S. Behavioral medicine revisited an amended definition. Journal of Behavioral Medicine, v. 1, p. 249-251, 1978.

18 SELIGMAN, M. E. P. Helplesness. San Francisco: Freeman, 1975.

19 SKINNER, B. F. Questões recentes na análise comportamental. Campinas: Papirus, 1991. 